Western University

Scholarship@Western

Brain and Mind Institute Researchers'

Publications

Brain and Mind Institute

$1-1-2012$

\title{
Discriminating famous from fictional names based on lifetime experience: evidence in support of a signal-detection model based on finite mixture distributions.
}

\author{
Ben Bowles \\ Western University \\ lain M Harlow \\ The University of Edinburgh \\ Melissa M Meeking \\ University of Western Ontario \\ Stefan Köhler \\ University of Western Ontario and Baycrest Centre
}

Follow this and additional works at: https://ir.lib.uwo.ca/brainpub

Part of the Neurosciences Commons, and the Psychology Commons

Citation of this paper:

Bowles, Ben; Harlow, lain M; Meeking, Melissa M; and Köhler, Stefan, "Discriminating famous from fictional names based on lifetime experience: evidence in support of a signal-detection model based on finite mixture distributions." (2012). Brain and Mind Institute Researchers' Publications. 243.

https://ir.lib.uwo.ca/brainpub/243 


\title{
Discriminating Famous From Fictional Names Based on Lifetime Experience: Evidence in Support of a Signal-Detection Model Based on Finite Mixture Distributions
}

\author{
Ben Bowles \\ University of Western Ontario \\ Melissa M. Meeking \\ University of Western Ontario
}

\author{
Iain M. Harlow \\ University of Edinburgh \\ Stefan Köhler
}

University of Western Ontario and Baycrest Centre

\begin{abstract}
It is widely accepted that signal-detection mechanisms contribute to item-recognition memory decisions that involve discriminations between targets and lures based on a controlled laboratory study episode. Here, the authors employed mathematical modeling of receiver operating characteristics (ROC) to determine whether and how a signal-detection mechanism contributes to discriminations between moderately famous and fictional names based on lifetime experience. Unique to fame judgments is a lack of control over participants' previous exposure to the stimuli deemed "targets" by the experimenter; specifically, if they pertain to moderately famous individuals, participants may have had no prior exposure to a substantial proportion of the famous names presented. The authors adopted established models from the recognition-memory literature to examine the quantitative fit that could be obtained through the inclusion of signal-detection and threshold mechanisms for two data sets. They first established that a signal-detection process operating on graded evidence is critical to account for the fame judgment data they collected. They then determined whether the graded memory evidence for famous names would best be described with one distribution with greater variance than that for the fictional names, or with two finite mixture distributions for famous names that correspond to items with or without prior exposure, respectively. Analyses revealed that a model that included a d' parameter, as well as a mixture parameter, provided the best compromise between number of parameters and quantitative fit. Additional comparisons between this equal-variance signal-detection mixture model and a dual-process model, which included a high-threshold process in addition to a signal-detection process, also favored the former model. In support of the conjecture that the mixture parameter captures participants' prior experience, the authors found that it was increased when the analysis was restricted to names in occupational categories for which participants indicated high exposure.
\end{abstract}

Keywords: criteria, fame judgments, receiver operating characteristic, recognition memory, signaldetection theory

One of the most elementary ways to probe declarative long-term memory is to examine the ability to recognize stimuli that have been encountered previously. A large body of research has been

This article was published Online First September 12, 2011.

Ben Bowles and Melissa M. Meeking, Department of Psychology, University of Western Ontario, London, Ontario, Canada; Iain M. Harlow, Neuroinformatics Doctoral Training Centre, School of Informatics, University of Edinburgh, Edinburgh, Scotland; Stefan Köhler, Department of Psychology, University of Western Ontario, London, Ontario, Canada, and Rotman Research Institute, Baycrest Centre, Toronto, Ontario, Canada.

This research was supported by the Natural Sciences and Engineering Research Council of Canada, through a graduate scholarship to Ben Bowles and an operating grant to Stefan Köhler. We thank Andy Yonelinas, Lawrence DeCarlo, Ken Malmberg, and Colleen Parks for their thoughtful comments.

Correspondence concerning this article should be addressed to Ben Bowles, Department of Psychology, University of Western Ontario, Social Science Center, 1151 Richmond Street, London, ON N6A5C2. E-mail: bbowles2@uwo.ca conducted with an attempt to characterize the discrimination processes involved in recognition-memory experiments using receiver operating characteristics (ROC; for recent reviews, see Wixted, 2007a; Yonelinas \& Parks, 2007). Participants are typically presented with a set of target items in a study phase and are later asked to discriminate between these items and novel intermixed lures in a test phase. Although ROC data can be gleaned from such paradigms in many ways, most commonly, participants must rate their confidence that each item was, or was not, encountered in the earlier study phase on a graded scale, with each response option reflecting a different response criteria. Debate regarding which model of discrimination processes best accounts for ROC data from recognition-memory experiments has been active, and sometimes heated, since the first mathematical models were developed more than 50 years ago (e.g., Egan, 1958). Today, the extant models can be grouped into those that rely on signal-detection mechanisms, threshold assumptions, or a hybrid of both (Yonelinas \& Parks, 2007); further, these models differ in terms of whether they assume one or more than one retrieval process. 
Signal-detection models assume that targets and lures have graded memory strength and are represented by overlapping Gaussian distributions (Green \& Swets, 1966; Macmillan \& Creelman, 2005; Wickens, 2002). Although there is no unanimous agreement (e.g., Bröder \& Schütz, 2009), most researchers agree that threshold mechanisms by themselves are insufficient to account for item-recognition memory and that any successful model requires the inclusion of signal-detection mechanisms.

The purpose of the current article is to examine the discrimination processes involved in recognition outside the laboratory, which includes situations such as perceiving a name or a face of a famous person as familiar. In past research, it has often been assumed that recognition based on a discrete study episode in item-recognition memory paradigms provides a means to model recognition that arises out of a lifetime of experience (Atkinson \& Juola, 1974; Mandler, 1980). The recognition-memory paradigm is clearly a convenient means to study recognition processes, as it permits precise experimental control over participants' exposure to the target stimuli and references a specific study episode at the retrieval stage. However, for this very reason it may not be particularly well-suited to model real-life recognition decisions that are not tied to a controlled, discrete study episode but, instead, to potentially multiple episodes, which participants may or may not be able to recollect and which may remain temporally undefined to them. Although some cognitive theories explicitly postulate similarities in mechanisms between these two types of situations (e.g., the SAC model; Diana, Reder, Arndt, \& Park, 2006; Reder et al., 2000), the extent to which they are indeed similar in terms of discrimination processes has largely been unexamined. Most important, perhaps, it is even unclear whether the most basic aspect of decisions in item-recognition memory experiments, namely, that they are supported by an underlying memory signal that is graded in nature, also characterizes recognition decisions made outside the laboratory.

To investigate the discrimination processes involved in everyday recognition, we presented participants with a selected set of moderately famous names, intermixed with matched fictional names, and asked them to rate their confidence that each name referred to a famous person from the media. By modeling famous names as targets and fictional names as lures, we were able to examine the discrimination processes that differentiate famous from fictional names using the same analytical and statistical techniques employed in past research on recognition memory that involved a study phase in the laboratory. Specifically, we employed maximum-likelihood estimation to model our data with reference to well-established threshold and signal-detection discrimination mechanisms derived from the recognition-memory literature.

Inherent in the approach that employs fame judgments to probe real-life recognition is the notion that participants' life experience with the famous names (i.e., target stimuli) is reflected in their memory strength or familiarity, which provides the basis for discriminating them from nonfamous, fictional names (i.e., the lure stimuli). As a result, unlike in recognition-memory paradigms, where stimulus exposure is controlled, participants may never have had any exposure to some of the famous names deemed target stimuli by the experimenter. How might this lack of exposure be reflected in the distributions that represent memory evidence for famous names overall? Given that some of the famous names to which participants have had no exposure are likely to be associated with particularly low memory evidence compared with famous names with exposure, the variance in the distribution of evidence for famous names overall is likely to be greater than that for fictional names. This scenario could perhaps be captured through an unequal-variance signal-detection (UVSD) model, one of the more popular models in the recognition-memory literature (Wixted, 2007a). However, given that exposed and nonexposed items can be seen to reflect two distinct classes of target stimuli, it is likely that famous names may in fact be better described with two Gaussian distributions, rather than a single Gaussian distribution with greater variance. As famous names with no exposure are not associated with any specific memory evidence generated by prior experience, they should be represented with the same distribution of memory evidence as fictional names. In contrast, famous names with exposure should be represented as a distribution with increased memory strength.

To discern whether one distribution with greater variance or two separate distributions best describes the memory evidence for famous names, we first modeled the discrimination of famous from fictional names using a signal-detection model that includes both components. Specifically, the model we employed includes one parameter that defines the proportion of famous names associated with prior exposure and one parameter that defines the ratio between the variance of the famous name distribution with exposure and the variance of the distribution for fictional names. Mathematically, this full model can be described as an unequal variance signal-detection model with finite mixture distributions (henceforth labeled the UVSD mixture model; see Figure 1a and Table 1 for full model equations). The generalized equation for the proportion of endorsed famous names in this model is given by

$$
p \text { ("yes" } \leq k \mid \text { "famous") }=(1-\lambda) \Phi\left(c_{\mathrm{k}}, 0,1\right)+\lambda \Phi\left(c_{\mathrm{k}}, d^{\prime}, \sigma_{\mathrm{FAM}}\right)
$$

Here $\Phi$ denotes the Gaussian distribution function, $d^{\prime}$ represents the distance in memory strength between the distribution for famous names with exposure and that for fictional names, $\lambda$ denotes the proportion of famous names to which the participant has been exposed (ranging from 0 to 1$), \sigma_{\text {FAM }}$ represents the standard deviation of the famous name distribution with exposure (constrained to be greater than the fictional-name distribution, arbitrarily set to 1 ), and $c_{\mathrm{k}}$ is a memory-strength criterion set by the participant for each level of memory strength. The generalized equation for the proportion of endorsed fictional names in this model is given by

$$
p \text { ("yes" } \leq \mathrm{k} \mid \text { "fictional" })=\Phi\left(c_{\mathrm{k}}, 0,1\right)
$$

In the current model, when $\sigma_{\mathrm{FAM}}=1$, the variance of the famous-name distribution with exposure becomes equal in variance to the fictional-name distribution. It is worth noting that this two-parameter model, which we label the equal-variance signaldetection (EVSD) mixture model, has been suggested previously to account for recognition memory by DeCarlo (2002; see Discussion for further detail). Setting $\lambda=1$ in the UVSD mixture model yields the UVSD model, which some researchers favor as the most suitable model of recognition memory in the literature (e.g., Wixted, 2007a). Restricting both $\lambda=1$ and $\sigma_{\text {FAM }}=1$ yields the simplest signal-detection model, the EVSD model, which is often considered to be the most basic framework of signal-detection 


\section{A. UVSD Mixture}
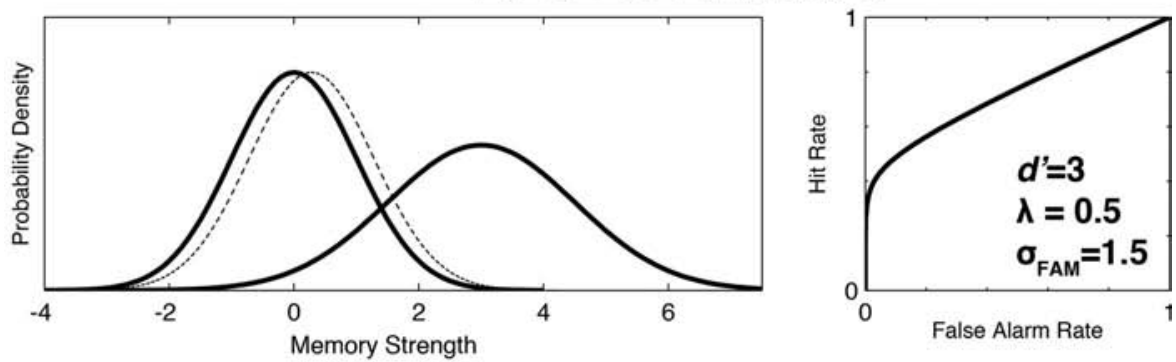

B. EVSD Mixture
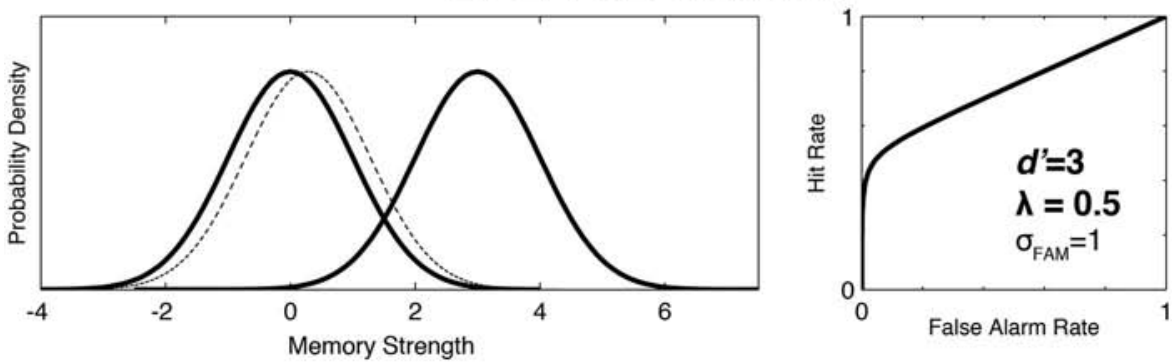

C. UVSD
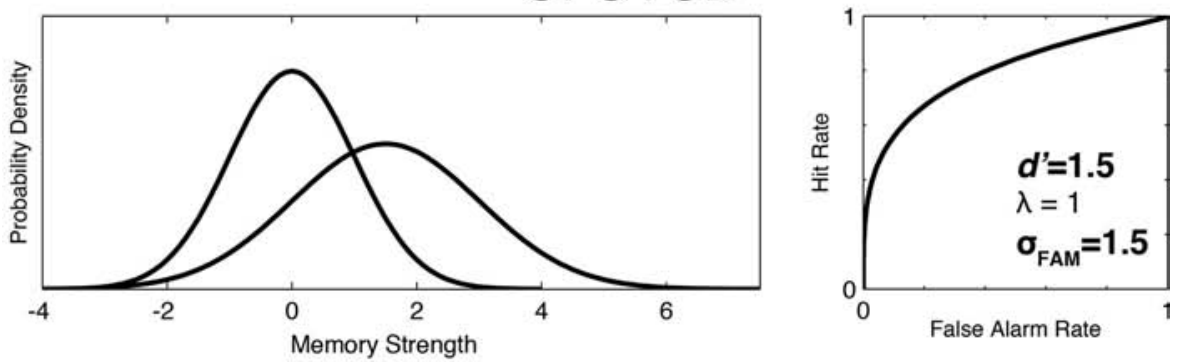

D. EVSD
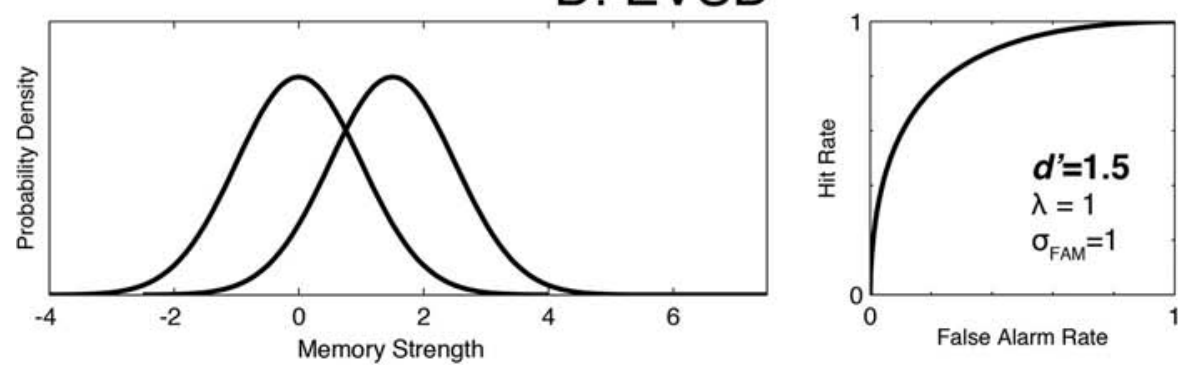

Figure 1. Visual illustration of the unequal-variance signal-detection (UVSD) mixture model and associated nested models in terms of Gaussian distributions and associated receiver operating characteristic plots. Values of freely varying parameters are indicated in bold and set for visual illustration only. The UVSD mixture model (A, full model) includes three freely varying theoretically relevant parameters $\left(d^{\prime}, \lambda\right.$, and $\sigma_{\text {FAM }}$ ). Setting $\sigma_{\text {FAM }}=1$ yields the equal-variance signal-detection (EVSD) mixture model (B), and setting $\lambda=1$ yields the UVSD model (C), respectively. Setting both $\sigma_{\mathrm{FAM}}=1$ and $\lambda=1$ yields the EVSD model (D). In A and B, the distribution of famous names with no exposure is depicted by a slightly offset broken line and has an identical mean strength and variance to the adjacent fictional-name distribution. 
Table 1

Equations for the UVSD Mixture Model

\begin{tabular}{c} 
Famous \\
$P(R=1)=(1-\lambda) * \Phi\left(c_{1}, 0,1\right)+\lambda * \Phi\left(c_{1}, d^{\prime}, \sigma_{\mathrm{FAM}}\right)$ \\
$P(R=2)=(1-\lambda) *\left[\Phi\left(c_{2}, 0,1\right)-\Phi\left(c_{1}, 0,1\right)\right]+\lambda *\left[\Phi\left(c_{2}, d^{\prime}, \sigma_{\mathrm{FAM}}\right)-\Phi\left(c_{1}, d^{\prime}, \sigma_{\mathrm{FAM}}\right)\right]$ \\
$P(R=3)=(1-\lambda) *\left[\Phi\left(c_{3}, 0,1\right)-\Phi\left(c_{2}, 0,1\right)\right]+\lambda *\left[\Phi\left(c_{3}, d^{\prime}, \sigma_{\mathrm{FAM}}\right)-\Phi\left(c_{2}, d^{\prime}, \sigma_{\mathrm{FAM}}\right)\right]$ \\
$P(R=4)=(1-\lambda) *\left[\Phi\left(c_{4}, 0,1\right)-\Phi\left(c_{3}, 0,1\right)\right]+\lambda *\left[\Phi\left(c_{4}, d^{\prime}, \sigma_{\mathrm{FAM}}\right)-\Phi\left(c_{3}, d^{\prime}, \sigma_{\mathrm{FAM}}\right)\right]$ \\
$P(R=5)=(1-\lambda) *\left[\Phi\left(c_{5}, 0,1\right)-\Phi\left(c_{4}, 0,1\right)\right]+\lambda *\left[\Phi\left(c_{5}, d^{\prime}, \sigma_{\mathrm{FAM}}\right)-\Phi\left(c_{4}, d^{\prime}, \sigma_{\mathrm{FAM}}\right)\right]$ \\
$P(R=6)=(1-\lambda) *\left[1-\Phi\left(c_{5}, 0,1\right)\right]+\lambda *\left[1-\Phi\left(c_{5}, d^{\prime}, \sigma_{\mathrm{FAM}}\right)\right]$ \\
\hline
\end{tabular}

Fictional

$$
\begin{aligned}
& p(R=1)=\Phi\left(c_{1}, 0,1\right) \\
& p(R=2)=\Phi\left(c_{2}, 0,1\right)-\Phi\left(c_{1}, 0,1\right) \\
& p(R=3)=\Phi\left(c_{3}, 0,1\right)-\Phi\left(c_{2}, 0,1\right) \\
& p(R=4)=\Phi\left(c_{4}, 0,1\right)-\Phi\left(c_{3}, 0,1\right) \\
& p(R=5)=\Phi\left(c_{5}, 0,1\right)-\Phi\left(c_{4}, 0,1\right) \\
& p(R=6)=1-\Phi\left(c_{5}, 0,1\right)
\end{aligned}
$$

Note. $\quad$ UVSD $=$ unequal-variance signal-detection. $p(R=\mathrm{i})$ denotes the probability of response category $i(i=$ $1,2, \ldots 6) ; \Phi$ denotes the cumulative Gaussian distribution function; $d^{\prime}$ denotes the separation in standard deviation units between the distribution for famous names with exposure and that for fictional names; $\lambda$ denotes the proportion of famous names to which the participant has been exposed; $\sigma_{\mathrm{FAM}}$ represents the standard deviation of the famous name distribution with exposure; and $c_{\mathrm{k}}$ is a memory strength criterion set by the participant for each level of memory strength.

theory (Green \& Swets, 1966; Macmillan \& Creelman, 2005; Wickens, 2002). Figure 1 illustrates these models in terms of Gaussian distributions and corresponding idealized ROC plots. Here we evaluated the fit of the proposed UVSD mixture model and compared it with its associated nested models: the EVSD mixture model, the UVSD model, and the EVSD model, with particular emphasis on the former two nested models, given the limited ability of the EVSD model to provide a good fit. Specifically, we examined the relative importance of the two most critical parameters of interest ( $\lambda$ and $\sigma_{\mathrm{FAM}}$ ) by comparing the full UVSD mixture model with the two models where each of these two specific parameters was restricted in isolation (i.e., the UVSD and EVSD mixture models, respectively).

Although the models discussed so far are limited to the inclusion of signal-detection mechanisms, some models in recognition memory, most notably the dual-process signal-detection model (DPSD), invoke both types of detection processes. The DPSD model includes two independent processes that contribute to discrimination: recollection, which is reflected as the proportion of recognized targets in the context of a single high-threshold discrimination process, and familiarity, which is reflected in an EVSD process (Yonelinas, 1994, 1999). Recollection is reflected in recognition associated with recall of contextual details, whereas familiarity is associated with recognition in the absence of such recall. The DPSD model has been closely compared with the UVSD model in the literature (e.g., Parks \& Yonelinas, 2007; Wixted, 2007b) and often provides comparable results at the level of quantitative fit. Given the popularity of the DPSD model, and given prior evidence suggesting that recollection can contribute to fame judgments (Piolino, Lamidey, Desgranges, \& Eustache, 2007; Westmacott \& Moscovitch, 2003), we also performed our analyses with a DPSD mixture model. In the DPSD mixture model that we employed, instead of allowing the famous-name distribution with exposure to have a greater variance than the fictional distribution, we allowed for the contribution of an independent high-threshold process. The generalized equation for the proportion of endorsed famous names in this model is given by

$$
\begin{aligned}
p(\text { "yes" } \leq & k \mid \text { "famous") } \\
& =T+(1-T) *\left[(1-\lambda) * \Phi\left(c_{1}, 0,1\right)+\lambda * \Phi\left(c_{1}, d, 1\right)\right]
\end{aligned}
$$

Here $T$ corresponds to recollection, or the proportion of famous names detected via a high-threshold process (fictional names identical to preceding full model; see Table 2 for full model equations). If one sets $\lambda=1$, then mathematically, the model collapses into the DPSD model; moreover, setting $T=0$ yields the EVSD mixture model previously described. By examining various nested models within this full model, we directly compared the importance of a mixture parameter $(\lambda)$ with a parameter that denotes the proportion of accurately recognized targets within the context of a high-threshold process $(T)$.

\section{Method}

\section{Participants}

Seventeen University of Western Ontario students (7 women, 10 men) with a mean age of 24.7 years (range $=18-32$ years) participated in the study and were compensated for their time. Two participants were removed from the analysis because they confidently recognized fewer than $10 \%$ of the famous names presented. The study received expedited research ethics approval in the Psychology Department at the University of Western Ontario.

\section{Materials}

Three hundred and five famous names were acquired from websites (e.g., www.canadians.ca, www.wikipedia.org, www.imdb.com). Celebrities were sampled from various nationalities, but we ensured that each of them had a high likelihood of some media 
Table 2

Equations for the DPSD Mixture Model

\begin{tabular}{c} 
Famous \\
$p(R=1)=T+(1-T) *\left[(1-\lambda) * \Phi\left(c_{1}, 0,1\right)+\lambda * \Phi\left(c_{1}, d^{\prime}, 1\right)\right]$ \\
$p(R=2)=(1-T) *\left\{\left[(1-\lambda) *\left(\Phi\left(c_{2}, 0,1\right)-\Phi\left(c_{1}, 0,1\right)\right]+\lambda *\left[\Phi\left(c_{2}, d^{\prime}, 1\right)-\Phi\left(c_{1}, d^{\prime}, 1\right)\right]\right\}\right.$ \\
$p(R=3)=(1-T) *\left\{\left[(1-\lambda) *\left(\Phi\left(c_{3}, 0,1\right)-\Phi\left(c_{2}, 0,1\right)\right]+\lambda *\left[\Phi\left(c_{3}, d^{\prime}, 1\right)-\Phi\left(c_{2}, d^{\prime}, 1\right)\right]\right\}\right.$ \\
$p(R=4)=(1-T) *\left\{\left[(1-\lambda) *\left(\Phi\left(c_{4}, 0,1\right)-\Phi\left(c_{3}, 0,1\right)\right]+\lambda *\left[\Phi\left(c_{4}, d^{\prime}, 1\right)-\Phi\left(c_{3}, d^{\prime}, 1\right)\right]\right\}\right.$ \\
$p(R=5)=(1-T) *\left\{\left[(1-\lambda) *\left(\Phi\left(c_{5}, 0,1\right)-\Phi\left(c_{4}, 0,1\right)\right]+\lambda *\left[\Phi\left(c_{5}, d^{\prime}, 1\right)-\Phi\left(c_{4}, d^{\prime}, 1\right)\right]\right\}\right.$ \\
$p(R=6)=(1-T) *\left\{\left[(1-\lambda) *\left(1-\Phi\left(c_{5}, 0,1\right)\right]+\lambda *\left[1-\Phi\left(c_{5}, d^{\prime}, 1\right)\right]\right\}\right.$ \\
\hline
\end{tabular}

Fictional

$$
\begin{aligned}
& p(R=1)=\Phi\left(c_{1}, 0,1\right) \\
& p(R=2)=\Phi\left(c_{2}, 0,1\right)-\Phi\left(c_{1}, 0,1\right) \\
& p(R=3)=\Phi\left(c_{3}, 0,1\right)-\Phi\left(c_{2}, 0,1\right) \\
& p(R=4)=\Phi\left(c_{4}, 0,1\right)-\Phi\left(c_{3}, 0,1\right) \\
& p(R=5)=\Phi\left(c_{5}, 0,1\right)-\Phi\left(c_{4}, 0,1\right) \\
& p(R=6)=1-\Phi\left(c_{5}, 0,1\right)
\end{aligned}
$$

Note. $\quad$ DPSD $=$ dual-process signal-detection. $T$ denotes the proportion of famous names endorsed within a probabilistic high-threshold process. All other parameters as per the unequal-variance signal-detection mixture model.

exposure in the country where the study was conducted (Canada). At the same time, names corresponding to individuals who would likely elicit confident recognition by every participant (e.g., Barack Obama) were avoided. Chosen famous names were sampled broadly from different categories, namely, business people (e.g., Jack Warner), comedians (e.g., Howie Mandel), models (e.g., Lauren Hutton), authors (e.g., Alice Munro), film actors (e.g., Meryl Streep), politicians (e.g., Michael Ignatieff), athletes (e.g., Ed Belfour), TV actors (e.g., Cynthia Nixon), musicians (e.g., Carrie Underwood), and people who did not fit clearly into any of the above categories (e.g., Roberta Bondar, Canada's first female astronaut). Using the Wikipedia online encyclopedia (www.wikipedia.org), we checked all names to ensure that they corresponded to people who became famous after World War II and were not well known in the media in association with a middle name (e.g., Billy Bob Thornton). Ninetyfive fictional names were created by randomly combining first and last names from the United States Census Bureau 1990 database (http://www.census.gov/genealogy/www/). Famous and fictional names were matched on the total number of letters and syllables and the sum frequency of first and last names based on information acquired from the U.S. Census database. We ensured that no fictional names inadvertently referred to famous names by verifying that the name was not associated with a specific entry in the Wikipedia online encyclopedia.

\section{Experimental Procedure}

Participants were told that they would view a list of names composed of approximately three quarters famous names and one quarter fictional names. It was made clear that famous names referred to the names of famous people that participants might have encountered in the media and that fictional names referred to random combinations of first and last names that did not refer to a publicly known individual. Famous and fictional names were presented to participants in random order one at a time in the center of a computer screen using E-Prime software (Psychology Software Tools; www.pstnet.com). Participants were required to make recognition decisions and to indicate their confidence in these decisions; using a computer keyboard, participants made their judgments by responding on a scale from 1 (sure the name is fictional) to 6 (sure the name is famous); responses 2-5 were used for intermediate degrees of confidence. Responses were given in a self-paced manner, and a sheet with a visual depiction of the response options was visible at all times during the experiment.

After completing the recognition-confidence ratings for all famous names, participants were asked to rate their relative degree of perceived day-to-day exposure to the nine different aspects of the media associated with the nine occupations listed above (e.g., "sports" for athletes). Specifically, participants were asked to rank order the different media domains on the basis of their perceived lifetime exposure.

\section{Experiment 1}

\section{Modeling Approach}

First we used maximum-likelihood estimation to fit each participant's data separately to various discrimination models derived from the recognition-memory literature. We concentrated on the examination of receiver operating characteristics (ROC) at the individual subject level based on research showing that artifacts can be introduced when ROC data are averaged (Malmberg \& Xu, 2006). Optimizations were performed using the "fminunc" function in MATLAB (Mathworks; www.mathworks.com), employing several different parameter starting values; optimizations were also validated using Excel Solver (Frontline Systems; www.solver.com). Additional visual examinations were conducted to ensure that each model fit matched each participant's empirical raw data (see Figure 2 for raw data and superimposed model fits obtained with the EVSD mixture model). For each fit, we minimized the negative log likelihood of the data $\left[-\Sigma N_{\mathrm{i}} \log p_{\mathrm{i}}\right]$, where $N_{\mathrm{i}}$ is the number of responses in category $i$ and $p_{\mathrm{i}}$ is the probability of response $i$ predicted by the model (see Ogilvie \& Creelman, 1968). 

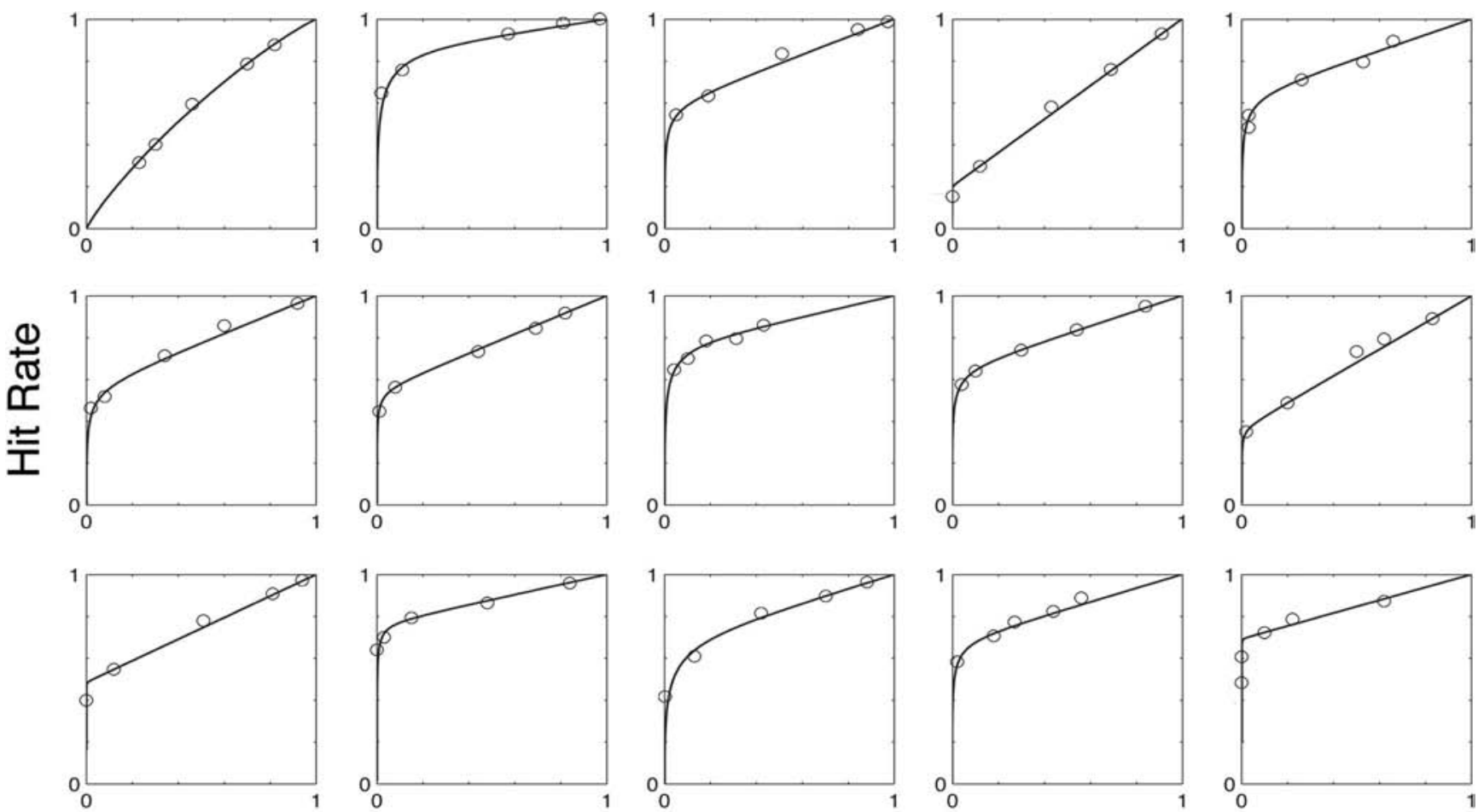

False Alarm Rate

Figure 2. Each participant's best fitting equal-variance signal-detection (EVSD) mixture model function superimposed on the raw receiver operating characteristic data from Experiment 1. Hit and false alarm rates reflect the proportions of famous and fictional names that exceed the memory strength designated by each of the five response criteria.

\section{Comparison of the Two High-Threshold Model With the UVSD Model}

We began by examining whether famous name recognition is supported by graded mnemonic evidence (i.e., a signaldetection process) or by purely discrete threshold mechanisms. Although the majority of the recognition memory ROCs examined in the literature are curvilinear, and thus preferentially support the notion that graded evidence supports recognition memory judgments, other investigators have argued that the extant research has not adequately ruled out threshold models such as the two high-threshold (2HT) model (e.g., Bröder \& Schütz, 2009; Erdfelder, Küpper-Tetzel, \& Mattern, 2011; Krantz, 1969; Malmberg, 2002). Thus, we compared the quantitative fit provided by the $2 \mathrm{HT}$ model, with that provided by the UVSD model. ${ }^{1}$ As the UVSD and 2HT models have the same number of model parameters and have been particularly well studied in the recognition-memory literature, they provide a good way to assess whether fame judgments are supported by signal-detection or threshold mechanisms. The $2 \mathrm{HT}$ model assumes two discrete memory states, represented by two separate model parameters that are constrained to vary between zero and one; targets can be in the "detect" state with some probability (Dt), and lures can be in a "reject" state with some other probability (Dl; Erdfelder et al., 2011; Macmillan, Rotello, \& Verde, 2005; Snodgrass \& Corwin, 1988). Target and lure items that are in neither of these two states are thus, by definition, in an indeterminate state and are endorsed as targets with a specific probability dependent on the level of bias applied by the participant. Thus, the model that we employed also includes five parameters for varying degrees of bias in addition to the two separate parameters for detecting targets and rejecting lures.

Mean parameter values as well as goodness-of-fit statistics are indicated in Table 3 . The results suggest that, for the $2 \mathrm{HT}$ model, $44 \%$ of famous names were in the "detect" state, whereas $7 \%$ of the fictional names were in the "reject" state. For the UVSD model, the results suggest that on average, the mean of the famous name distribution had a variance that was 1.81 times that of the fictional-name distribution, with a mean offset by 0.86 standard deviations from the fictional name distribution. Notably, the ratio of variances for famous-name targets compared with fictionalname lures is larger compared with the ratio of variances between

\footnotetext{
${ }^{1}$ We also explored whether the less commonly implemented single high-threshold model (Luce, 1963) may provide a satisfactory account. We rejected it, as it was deemed to provide an inferior fit compared with the 2HT account using all means of model comparisons. Nested likelihood ratio tests showed that the additional inclusion of the Dl parameter in the $2 \mathrm{HT}$ model statistically improved the fit of the single high-threshold model, $\chi^{2}(15)=37.04, p<.001$.
} 
Table 3

Comparison of the $2 H T$ With the UVSD Model

\begin{tabular}{|c|c|c|c|c|c|c|c|c|c|c|}
\hline \multirow[b]{3}{*}{ Model } & \multirow{2}{*}{\multicolumn{6}{|c|}{ Goodness-of-fit statistics }} & \multicolumn{4}{|c|}{ Estimated parameter values } \\
\hline & & & & & & & \multicolumn{2}{|c|}{$2 \mathrm{HT}$} & \multicolumn{2}{|c|}{ UVSD } \\
\hline & No. of parameters & $d f$ & $G^{2}$ & $p\left(G^{2}\right)$ & Sum AIC & Sum BIC & $M \mathrm{Dt}$ & $M \mathrm{Dl}$ & $M d^{\prime}$ & $M \sigma_{\mathrm{FAM}}$ \\
\hline \multicolumn{11}{|c|}{ Experiment 1} \\
\hline $2 \mathrm{HT}$ & 105 & 45 & 180.54 & $<0.001$ & $17,869.50$ & $18,204.76$ & 0.44 & 0.07 & & \\
\hline UVSD & 105 & 45 & 100.83 & $<0.001$ & $17,789.79$ & $18,125.05$ & & & 0.86 & 1.81 \\
\hline \multicolumn{11}{|c|}{ Experiment 2} \\
\hline $2 \mathrm{HT}$ & 84 & 36 & 366.97 & $<0.001$ & $14,932.54$ & $15,182.00$ & 0.35 & 0.08 & & \\
\hline UVSD & 84 & 36 & 67.63 & 0.02 & $14,633.20$ & $14,882.66$ & & & 0.87 & 1.91 \\
\hline
\end{tabular}

Note. $2 \mathrm{HT}=$ two high-threshold; UVSD = unequal-variance signal-detection; AIC = Akaike information criterion; BIC = Bayesian information criterion; Dt = "detect" state; D1 = "reject" state.

targets and lures in recognition memory (approximately 1.25 ; see Ratcliff, Sheu, \& Gronlund, 1992). To compare the UVSD model with the 2HT model directly, we first computed the $G^{2}$ statistic ${ }^{2}$ to examine the hypothesis that these models should be rejected (Sokal \& Rohlf, 1994). Examination of these values showed that the null hypothesis (i.e., the notion that the model provided an adequate fit of the data) was rejected for both the 2HT model, $\chi^{2}(30)=180.53, p<.001$, as well as the UVSD model, $\chi^{2}(30)=$ $100.83, p<.001$ (see Table 3). Although both models were rejected, it is worth noting that $G^{2}$ was numerically lower in the UVSD model compared with the 2HT model, suggesting that the former model provides the better fit.

Next, we calculated Akaike's Information Criterion (AIC; Akaike, 1974) and the Bayesian information criterion (BIC; Schwarz, 1978) for all individual fits. ${ }^{3}$ Both information criteria were found to be lower for the UVSD model, compared with the 2HT model, when values for all participants were summed, again pointing to a better fit of the former model (see Table 3). This pattern was also present on both measures in nine of the 15 individual participants examined. Thus, all measures converge in demonstrating the superiority of the UVSD model over the 2HT model in terms of quantitative fit. This result provides support for the notion that the discrimination processes involved in famousname recognition cannot be fully captured by a model that solely relies on threshold mechanisms, and by extension highlights the importance of including a process based on graded memory evidence.

\section{UVSD Mixture Model Analysis}

Next we fit the data with the UVSD mixture model, as described in the introduction, to determine whether one or two distributions best capture the underlying memory evidence for famous names that was shown to be graded in our initial set of analyses. The full UVSD mixture model involved solving for eight free parameters: five criteria and three theoretically relevant model parameters (see Figure $1 \mathrm{a} ; d^{\prime}, \lambda$, and $\sigma_{\mathrm{FAM}}$ ). The EVD mixture (see Figure $1 \mathrm{~b}$ ) model and the UVSD model (see Figure 1c) were obtained by separately restricting either $\sigma_{\mathrm{FAM}}=1$ or $\lambda=1$, respectively. The EVSD model was defined by having only one famous-name distribution with the same variance as the fictional-name distribution; thus, it corresponded to a model in which both $\sigma_{\mathrm{FAM}}=1$ and $\lambda=$ 1. Testing of the four model fits using the $G^{2}$ statistic showed that the null hypothesis (that the model fit the data) was rejected for the EVSD model, $\chi^{2}(60)=469.74, p<.001$, the UVSD model, $\chi^{2}(45)=$ $100.83, p<.001$, and the UVSD mixture model, $\chi^{2}(30)=47.57, p<$ .05 , but not for the EVSD mixture model, $\chi^{2}(45)=57.34, p=.10$ (see Table 4). Table 4 shows goodness-of-fit statistics across participants for all four models examined.

Examination of the AIC and BIC at the level of fits for individual participants revealed that the EVSD mixture model was the best fit in 13 out of 15 participants for both measures. The AIC and the BIC were also lowest for the EVSD mixture model when the data were summed across participants. This provides further evidence to suggest that this model provides the best compromise between quantitative fit and number of parameters.

Given that the quantitative fit of the UVSD model and the EVSD mixture model are reasonably similar, we investigated in another way which of these two models should be considered more appropriate. Specifically, we used log-likelihood ratio tests ${ }^{4}$ to examine the relative statistical importance of the mixture parameter versus the ratio variance parameter in describing the current data. We compared the fit of the full UVSD mixture model, which

\footnotetext{
${ }^{2}$ The $G^{2}$ statistic is defined by $\left[2 \sum O_{\mathrm{ij}} \log \left(O_{\mathrm{ij}} / E_{\mathrm{ij}}\right)\right]$ and is well fit by a chi-squared distribution. The $G^{2}$ has been shown to be a more suitable goodness-of-fit statistic than the similar chi-squared statistic (Sokal \& Rohlf, 1994). In all analyses that we report, statistics for the chi-squared test were also examined but did not differ in any considerable way from the $G^{2}$ statistics we report, neither in value nor in terms of significance.

${ }^{3}$ The AIC and the BIC take into consideration the estimated log likelihood and the number of free parameters in each model and thus provide a relative gauge of the suitability of many comparable models; the model with the lowest value should be preferred. Although both statistics involve a penalty for a larger number of parameters, the penalty for additional parameters is larger for BIC. As both the 2HT model and the UVSD model have the same number of parameters, similar comparative information could be gleaned simply be examining the minimized negative log likelihood values themselves. We include values of AIC and BIC for purpose of comparison with subsequently described models.

${ }^{4}$ To compare models, we performed nested likelihood ratio tests, defined by $\mathrm{D}=-2[\log ($ likelihood for null model $)-\log ($ likelihood for
} 
Table 4

Goodness-of-Fit Statistics and Estimated Parameters for the UVSD Mixture Model Analysis for Both Experiments

\begin{tabular}{|c|c|c|c|c|c|c|c|c|c|}
\hline \multirow[b]{2}{*}{ Model } & \multicolumn{6}{|c|}{ Goodness-of-fit statistics } & \multicolumn{3}{|c|}{ Estimated parameter values } \\
\hline & No. of parameters & $d f$ & $G^{2}$ & $p\left(G^{2}\right)$ & Sum AIC & Sum BIC & $M d^{\prime}$ & $M \lambda$ & $M \sigma_{\mathrm{FAM}}$ \\
\hline \multicolumn{10}{|c|}{ Experiment 1} \\
\hline EVSD & 90 & 60 & 469.74 & $<0.001$ & $18,128.70$ & $18,416.07$ & 1.01 & 1.00 & 1.00 \\
\hline EVSD Mix & 105 & 45 & 57.37 & 0.10 & $17,746.33$ & $18,081.59$ & 3.59 & 0.59 & 1.00 \\
\hline UVSD & 105 & 45 & 100.83 & $<0.001$ & $17,789.79$ & $18,125.05$ & 0.86 & 1.00 & 1.81 \\
\hline UVSD Mix & 120 & 30 & 47.57 & 0.02 & $17,766.54$ & $18,149.69$ & 1.69 & 0.66 & 1.83 \\
\hline \multicolumn{10}{|c|}{ Experiment 1: High-exposure analysis } \\
\hline EVSD & 90 & 60 & 332.75 & $<0.001$ & $8,728.68$ & $9,016.04$ & 1.33 & 1.00 & 1.00 \\
\hline EVSD Mix & 105 & 45 & 44.49 & 0.49 & $8,470.41$ & $8,805.67$ & 3.73 & 0.68 & 1.00 \\
\hline UVSD & 105 & 45 & 69.87 & 0.01 & $8,495.80$ & $8,831.06$ & 1.05 & 1.00 & 1.94 \\
\hline UVSD Mix & 120 & 30 & 36.94 & 0.18 & $8,492.87$ & $8,876.02$ & 2.06 & 0.74 & 1.68 \\
\hline \multicolumn{10}{|c|}{ Experiment 2} \\
\hline EVSD & 72 & 48 & 376.24 & $<0.001$ & $14,917.81$ & $15,131.64$ & 1.05 & 1.00 & 1.00 \\
\hline EVSD Mix & 84 & 36 & 35.48 & 0.84 & $14,601.04$ & $14,850.51$ & 3.72 & 0.55 & 0.00 \\
\hline UVSD & 84 & 36 & 67.63 & 0.02 & $14,633.20$ & $14,882.66$ & 0.87 & 1.00 & 1.91 \\
\hline UVSD Mix & 96 & 24 & 23.44 & 0.80 & $14,613.01$ & $14,898.11$ & 1.59 & 0.66 & 2.30 \\
\hline
\end{tabular}

Note. $\quad \mathrm{UVSD}=$ unequal-variance signal-detection; $\mathrm{AIC}=$ Akaike information criterion; $\mathrm{BIC}=$ Bayesian information criterion; $\mathrm{EVSD}=\mathrm{equal}-\mathrm{variance}$ signal-detection. Parameter estimates in bold indicate freely varying parameters.

includes $d^{\prime}, \lambda$, and $\sigma_{\mathrm{FAM}}$ as freely varying parameters, with both the fit of the EVSD mixture model and the UVSD mixture model, which included only $d^{\prime}$ and either $\lambda$ or $\sigma_{\mathrm{FAM}}$, respectively. Using these two comparisons, we separately assessed the relative importance of these two latter parameters to the fit of the full model. The full UVSD mixture model was a significant improvement over the UVSD model, $\chi^{2}(15)=53.25, p<.001$, but not a significant improvement over the EVSD mixture model, $\chi^{2}(15)=9.79 p=$ 83. In other words, even when the variance of the famous-name distribution was already allowed to be greater than that of the fictional-name distribution, the introduction of a second, separate distribution for famous names (with the same mean and variance as the fictional distribution) significantly improved the model fit. In contrast, when the mixture parameter $\lambda$ was already included as a freely varying parameter in the model, the introduction of an additional parameter that allowed the famous-name distribution with prior exposure to have a greater variance than the fictional-name distribution did not significantly improve the model fit.

\section{Analyses of $z$-ROCs}

Next, we examined the linearity of the ROC data plotted in $z$-space. In these analyses, we used the correction recommended by

alternative model)]. This test statistic is well described by a chi-squared distribution with degrees of freedom corresponding to the difference in parameters between the two models compared. Note that in those likelihood ratio tests that we report here, the simpler model is defined on the basis of a parameter that is fixed on the boundary of the parameter space (ranging between zero and one) in the more complex model to which it is compared. Some caution should apply when interpreting $p$ values from nested likelihood ratio tests when this is the case; research indicates that $p$ values yielded from such tests may be more conservative than their true values (Self \& Liang, 1987).
Snodgrass and Corwin (1988) to correct for undefined values caused by zero counts for a given confidence level in a given stimulus class. Whereas both the EVSD and UVSD models predict linear $z$-ROCs, models with finite mixture distributions can accommodate curvilinear $z$-ROCs as well (see DeCarlo, 2002). We fitted the five points on each participant's $z$-ROC curve to a quadratic equation and examined the quadratic coefficients $(\beta)$ for all participants individually. On average, quadratic parameters were statistically above zero indicating slightly concave $z$-ROCs, mean $\beta=0.055, t(14)=3.32, p<.01$. As other types of discrimination models can result in curvilinear $z$-ROCs (e.g., the DPSD model; see Yonelinas, 1994, 1999), we cannot claim that this reflects the specific presence of mixture distributions in the data. However, it provides additional evidence that neither the UVSD model nor the EVSD model can adequately describe the data, given that both models predict strictly linear $z$-ROCs.

\section{Dual-Process Signal-Detection Mixture Model Analysis}

Another influential model in the recognition-memory literature is the DPSD model developed by Yonelinas (1994, 1999). This model posits that recognition is best described by two independent processes, namely, familiarity and recollection. Like the UVSD model, the DPSD model employs a $d^{\prime}$ parameter corresponding to the distance in $z$-coordinates between target and lure distributions (corresponding to familiarity). However, the DPSD model invokes a parameter representing the proportion of recollected items in the context of a high-threshold process instead of a parameter representing the difference in variances between target and lure distributions (as in the UVSD model). Because the DPSD model implements a threshold function, it could also account for the asymmetry observed in the present ROCs, which appear to exhibit a strong linear component based on visual examination (see Figure 2). Moreover, the model also predicts curvilinearity in $z$-space for 
recognition-memory decisions. The asymmetry in native space and the curvilinearity in $z$-space that we observed in the current data may point to the contribution of a high-threshold process, a notion that would be in line with previous research suggesting that a recollective process contributes to the recognition of famous names based on lifetime exposure (Piolino et al., 2007; Westmacott \& Moscovitch, 2003).

Thus, we also examined a DPSD mixture model that included parameters for $d^{\prime}, \lambda$, and a high-threshold parameter $T$ (see Table 2 for complete model equations). By comparing various nested models within this full model, we aimed to determine whether the DPSD model might be a more suitable alternative than the EVSD mixture model to account for the presently acquired data. Moreover, using this modeling approach, we explored whether evidence in favor of a threshold process would emerge in the context of famous-name recognition when a parameter to account for lack of exposure is already included in the model. Setting either $T=0$ or $\lambda=1$ in the DPSD mixture model yields the EVSD mixture model or the DPSD model, respectively; restricting both parameters in this way at the same time results in the EVSD model. Table 5 shows goodness-of-fit statistics across participants for all four models examined. Testing of the four model fits using the $G^{2}$ statistic showed that the null hypothesis (that the model fit the data) was rejected for the EVSD model, $\chi^{2}(60)=469.74, p<$ .001 , and the DPSD model, $\chi^{2}(45)=92.95, p<.001$, but not for the EVSD mixture model, $\chi^{2}(45)=57.37, p=.10$, or the DPSD mixture model, $\chi^{2}(30)=43.57, p<.052$. The EVSD mixture model was considered most suitable based on examination of AIC and BIC when all participants were considered together (see Table 5 ) and in the majority of participants when considered individually (8/15). The DPSD mixture model was a significant improvement over the DPSD model, $\chi^{2}(15)=49.38, p<.001$, but, critically, not over the EVSD mixture model, $\chi^{2}(15)=13.80, p=.54$. From a statistical modeling perspective, this pattern of results points to the necessity of including the mixture parameter, but not the high-threshold parameter, in accounting for the current data.

\section{Exposure Analysis}

Within the context of our approach, it is assumed that the $\lambda$ parameter in the UVSD mixture and EVSD mixture models represents the proportion of famous names that participants have had some exposure to in their lifetime. If this assumption is correct, $\lambda$ should increase when participants have had lifetime exposure to a greater proportion of the famous names presented. Our rank-order data on self-rated exposure to the various media domains provides a means to test this notion. Thus, we performed our analysis again for the best fitting EVSD mixture model, including all fictional names but only those famous names in the two occupational categories for which participants indicated highest day-to-day exposure. In other words, this analysis included famous names specifically selected for each participant based on their individual occupation-exposure ratings. With the criteria specified, we selected on average 101.80 famous names for each participant (minimum $=82$, maximum $=116$ ).

Analyses based on a paired $t$ test revealed that the mean $\lambda$ was significantly greater $(\lambda=0.68)$ in the analysis that only included high-exposure items than the one that included the entire set of famous names, $\lambda=0.59, t(14)=6.44, p<.001$ (see Figure 3). That $\lambda$ increases when the analysis is limited to high-exposure famous names is consistent with our interpretation that it reflects the proportion of famous names that participants have encountered in their lifetime. Notably, when we compared the fit of the full UVSD mixture model with its associated nested models only for high-exposure items, the analysis also favored the same model (i.e., the EVSD mixture model) that emerged as the best fit when analyses included all items (see Table 4). The AIC and the BIC were the lowest for the EVSD mixture model, and this was the case for 10 of the 15 participants examined. Similar to the analysis that included all famous names, the full UVSD mixture model was a significant improvement over the UVSD model, $\chi^{2}(15)=32.93$, $p<.005$, but not a significant improvement over the EVSD mixture model, $\chi^{2}(15)=7.54, p=.94$.

Table 5

Goodness-of-Fit Statistics and Estimated Parameters for the DPSD Mixture Model Analysis for Both Experiments

\begin{tabular}{|c|c|c|c|c|c|c|c|c|c|}
\hline \multirow[b]{2}{*}{ Model } & \multicolumn{6}{|c|}{ Goodness-of-fit statistics } & \multicolumn{3}{|c|}{ Estimated parameter values } \\
\hline & No. of parameters & $d f$ & $G^{2}$ & $p\left(G^{2}\right)$ & Sum AIC & Sum BIC & $M d^{\prime}$ & $M \lambda$ & $M \mathrm{~T}$ \\
\hline \multicolumn{10}{|c|}{ Experiment 1} \\
\hline EVSD & 90 & 60 & 469.74 & $<0.001$ & $18,128.70$ & $18,416.07$ & 1.01 & 1.00 & 0.00 \\
\hline EVSD Mix & 105 & 45 & 57.37 & 0.10 & $17,746.33$ & $18,081.59$ & 3.59 & 0.59 & 0.00 \\
\hline DPSD & 105 & 45 & 92.95 & $<0.001$ & $17,781.91$ & $18,117.17$ & 0.36 & 1.00 & 0.43 \\
\hline DPSD Mix & 120 & 30 & 43.57 & 0.05 & $17,762.53$ & $18,145.68$ & 1.66 & 0.47 & 0.32 \\
\hline \multicolumn{10}{|c|}{ Experiment 2} \\
\hline EVSD & 72 & 48 & 376.24 & $<0.001$ & $14,917.81$ & $15,131.64$ & 1.05 & 1.00 & 0.00 \\
\hline EVSD Mix & 84 & 36 & 35.48 & 0.84 & $14,601.04$ & $14,850.51$ & 3.72 & 0.55 & 0.00 \\
\hline DPSD & 84 & 36 & 134.51 & $<0.001$ & $14,700.08$ & $14,949.55$ & 0.58 & 1.00 & 0.35 \\
\hline DPSD Mix & 96 & 24 & 22.72 & 0.83 & $14,612.29$ & $14,897.39$ & 2.82 & 0.45 & 0.28 \\
\hline
\end{tabular}

Note. $\quad$ DPSD $=$ dual-process signal-detection; AIC $=$ Akaike information criterion; $\mathrm{BIC}=$ Bayesian information criterion; EVSD $=$ equal-variance signal-detection. Parameter estimates in bold indicate freely varying parameters. 


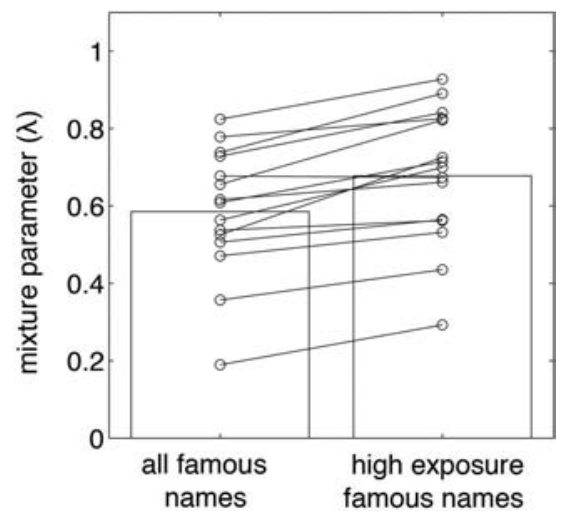

Figure 3. Quantitative estimates of the mixture parameter $(\lambda)$ for all participants in Experiment 1 for the analysis that included all famous names and the one that included only the famous names in the two occupational categories for which participants indicated highest day-to-day exposure.

\section{Experiment 2}

To determine whether our modeling conclusions would generalize to another data set obtained with the same task, we collected data from another set of 12 participants. Comparing the UVSD model with the $2 \mathrm{HT}$ model, the UVSD model again provided the superior fit overall (see Table 3). Subject-by-subject analyses of AIC and BIC revealed that these estimates were lower for the UVSD model compared with the 2HT model overall and in nine of the 12 participants tested. In the UVSD mixture analysis, we found that both the EVSD mixture model and the UVSD mixture models were considered acceptable fits of the data using the $G^{2}$ statistic (see Table 4). As in Experiment 1, both the AIC and the BIC were lowest for the EVSD mixture model, and this was the case for nine of the 12 participants when examined individually. Similar to our first sample, we observed, using likelihood ratio tests, that the UVSD mixture model offered a significant improvement over the UVSD model, $\chi^{2}(12)=44.18, p<.001$, but not a significant improvement over the EVSD mixture model, $\chi^{2}(12)=12.03, p=$ .44 .

In the DPSD mixture analysis, using the $G^{2}$ statistic, we found that both the EVSD mixture and the DPSD mixture models were considered acceptable fits of the data (see Table 5). Both the AIC and the BIC were lowest for the EVSD mixture model, and this was the case for 10 of the 12 participants examined individually. Similar to our first sample, we observed that the DPSD mixture model was a significant improvement over the DPSD model, $\chi^{2}(12)=111.80, p<.001$, but not a significant improvement over the EVSD mixture model, $\chi^{2}(12)=12.76, p=.39$. In line with our previous experiment, these results suggest that the EVSD mixture model is the most suitable signal-detection model to capture discriminations between famous and fictional names.

\section{Discussion}

In this study, we employed mathematical modeling of ROC data to characterize the discrimination processes that support the recognition of famous names based on lifetime experience. For this purpose, we adopted established models from the recognition- memory literature that included signal-detection and threshold mechanisms. We first compared a popular threshold model, the 2HT model, with the UVSD model and found evidence in support of the latter. Given that these two models are particularly wellstudied examples of pure threshold and pure signal-detection models in the recognition memory literature, these results thus argue in favor of graded underlying memory evidence rather than discrete retrieval states in fame judgments. We then explored whether the graded distribution of memory evidence for famous names would be best described with one distribution with greater variance than that for the fictional names or with two finite mixture distributions for famous names that correspond to items with and without prior exposure. To discern between these two possibilities, we fit our data with a model that incorporated a mixture parameter that reflected the proportion of famous names with exposure, as well as a parameter that reflected the ratio between the variance of the distribution for famous names with exposure and that for fictional names (i.e., the UVSD mixture model). We compared this full model with two nested models in which each of these two parameters was restricted separately, yielding the UVSD model and the EVSD mixture model, respectively. Examination of likelihood ratios, analyses of Akaike and Bayesian information criteria, and regression analyses of $z$-transformed ROC data revealed that the EVSD mixture model provided the best compromise between number of parameters and quantitative fit. Additional comparisons with a separate DPSD mixture model, which included a highthreshold parameter instead of a parameter for unequal variances, also favored the EVSD mixture model. After including the discrimination parameter and the mixture parameter in our signaldetection model, no other statistical parameters (i.e., neither $\sigma_{\mathrm{FAM}}$ in the case of the UVSD mixture model, nor $T$ in the case of the DPSD mixture model) led to statistical improvement in model fit.

To our knowledge, the present findings provide the first demonstration that recognition of famous names based on past life experience involves a discrimination process that operates on graded memory evidence (i.e., a signal-detection mechanism). Although there seems to be a broad consensus in the recognitionmemory literature that a signal-detection process contributes to recognition based on a discrete study episode, the application of these principles to recognition discriminations based on prior exposure outside the laboratory has received little attention in psychological research so far. Investigations in other domains of cognitive psychology, however, have recently begun to adopt this methodology for related questions involving other types of recognition judgments. For example, it has been shown that lexical decision judgments made in response to words presented for only $30 \mathrm{~ms}$ are supported by an equal-variance signal-detection mechanism, which is assumed to reflect a fast-acting familiarity process that can be dissociated from the word identification process that takes place in later stages (Jacobs, Graf, \& Kinder, 2003; see also Brown \& Steyvers, 2005; Paap, Chun, \& Vonnahme, 1999). Similarly, other research has shown that recognizing letter strings from a previously learned artificial grammar is also best described purely in terms of signal-detection mechanisms and underlying graded evidence (Kinder \& Assmann, 2000).

Although the majority of studies on recognition memory interpret the curvilinearity of ROCs generated with confidence judgments within a signal-detection framework, some investigators have argued that threshold models in combination with suitable 
response mappings can also produce curvilinear ROCs under these circumstances (Krantz, 1969; Larkin, 1965; Malmberg, 2002; see also Erdfelder et al., 2011). This type of concern may also be raised when interpreting the present data, as they involved confidence judgments. However, a threshold account of curvilinear ROCs along the lines mentioned has been criticized for lack of parsimony (Hilford, Glanzer, Kim, \& DeCarlo, 2002). In addition, recognition-memory ROCs generated with bias manipulations, rather than confidence ratings, have also yielded curvilinear ROCs in many cases (Fortin, Wright, \& Eichenbaum, 2004; Ratcliff et al., 1992). Given that, according to a threshold model, recognition judgments should always generate linear ROCs when based on bias manipulations (Malmberg, 2002), these results converge with confidence-rating experiments in supporting signal-detection mechanisms instead. In keeping with these arguments, we interpret the current results as strong support for the signal-detection framework that is favored in the field at large (Wixted, 2007a; Yonelinas \& Parks, 2007).

More specifically, the signal-detection framework also provides an intuitive and parsimonious way to understand differences at the level of ROCs between the judgments made in recognitionmemory experiments versus those made in the famous-name recognition task employed here. Critically, the two situations differ with respect to what specific type of signal-detection model should be considered most favorable. Unique to famous-name recognition is a lack of control over participants' previous exposure to the stimuli deemed "targets" by the experimenter. One consequence is that recognition may be tied to any number of life events, which are temporally undefined to the participant. Another consequence is that participants may, in fact, have had no life exposure to a proportion of the target items in the experiment, in effect making them indistinguishable from fictional names from the participants' point of view. This latter aspect of the current recognition task was successfully captured in the modeling approach employed here by implementing a mixture parameter that allowed a proportion of famous names to have the same memory-strength distribution as fictional names. Indeed, the results of our modeling analyses reveal that the mixture parameter, which we take to reflect the proportion of famous names associated with prior exposure, was necessary in that it consistently added to the model fit when compared with an otherwise identical model. In contrast, it is unclear how pure threshold mechanisms could account for the impact of exposure just described. With respect to the $2 \mathrm{HT}$ model, for example, although one can postulate distinct memory states for exposed and unexposed famous names, it remains unclear what process would allow an individual to determine which fictional names would fall into the "reject" compared with the indeterminate state.

The shapes of the individual participant ROCs, as illustrated in Figure 2, merit further discussion as well. The current ROCs appear more asymmetrical and linear than ROCs typically gleaned from recognition-memory experiments (see Parks \& Yonelinas, 2007, for a review). This difference cannot be explained based on the use of famous names as stimuli, given that item-recognition memory experiments with famous names as stimuli have also yielded ROCs that are more curvilinear than those currently observed (e.g., Stenberg, Hellman, \& Johansson, 2008). In itemrecognition memory, the observed asymmetry is often accounted for by invoking greater variance for the target distribution than for the lure distribution, as is the case for the UVSD model (for a review, see Wixted, 2007a). According to the dual-process equalvariance signal-detection model (DPSD), the asymmetry results from an independent high-threshold detection process, which supports the recollection of a certain proportion of studied targets (Yonelinas, 1994, 1999). In the model proposed by DeCarlo (2002), the asymmetry is evident because unattended items are represented in a separate distribution with identical mean and variance to that for the novel lures. This model is identical, in mathematical terms, to the EVSD mixture model that best captures the current data. In effect, the present EVSD mixture model treats famous names with or without exposure in the same way that DeCarlo's (2002) model treats targets that were attended or unattended at study. Thus, in the current implementation of the EVSD mixture model for famous-name recognition, we would argue that the current ROCs are particularly asymmetrical because a very large proportion of target famous names were never encountered.

Indeed, in the most favorable EVSD mixture model, estimates of the mixture parameter suggest that, on average, 0.41 of all famous names were associated with no life experience (i.e., $1-\lambda$; see Table 4). Such a high estimate is plausible within the context of our use of only moderately famous names from many different occupational categories, to which participants had varying degrees of exposure. In line with our interpretation of $\lambda$ as an index of prior exposure, when we restricted our analysis to include only famous names that were associated with those segments of the media to which participants had self-rated high exposure, the EVSD mixture model estimate for famous names without exposure dropped to 0.32 . The strategy that we employed to provide additional validity for $\lambda$ as an index of exposure (i.e., by restricting our analysis to high-exposure items) is similar to that employed by DeCarlo (2002) to describe the role of $\lambda$ as a measure of participants' attention in the study phase of recognition memory experiments. In that article, it was observed that certain variables predicted to have positive influences on attention, such as longer presentation time, are associated with increases in $\lambda$, similar to the currently observed effect of occupation exposure.

Other studies also point to a role for finite mixture distributions in recognition memory. Most notably, Sherman, Atri, Hasselmo, Stern, and Howard (2003) proposed a modification of the DPSD model, the variable recollection dual-process (VRDP) model, which postulates two separate Gaussian distributions, each with a freely varying mean and variance, for familiarity evidence and for recollection evidence. In subsequent developments of the VRDP model, the variances of all distributions were set to one, and only the means of the familiarity and recollection distributions were allowed to vary (Onyper, Zhang, \& Howard, 2010). This latter VRDP model is mathematically very similar to the currently proposed EVSD mixture model. Our model differs in that only one target distribution (i.e., for famous names with exposure) varies in mean memory evidence; the other distribution (i.e., for famous names without exposure) is fixed to be equal in mean and in variance to the fictional name distribution. This latter modeling decision was theoretically motivated; as we proposed that famous names without exposure should be identical to fictional names from the participants' point of view, we predicted they would be best described with the same Gaussian distribution. In support of this hypothesis, additional analyses revealed no statistical benefit 
from allowing the nonexposed famous name distribution to have a mean greater than zero.

It is also worth noting that finite mixture distributions have been employed in memory decisions other than those pertaining to item-recognition memory. For example, DeCarlo (2003, 2008) proposed that source decisions can also be described accurately with finite mixture distributions, if one considers that some source information may be either available or unavailable. An interesting commonality in the findings of DeCarlo (2008) and those reported here is that unequal variances among separate Gaussian distributions seem to be unnecessary once a mixture parameter is included in the fitted model. Moreover, finite mixture models have also been used to account for associative recognition, in which participants are required to discriminate between intact and rearranged pairs of stimuli (e.g., word pairs). For example, Kelley and Wixted (2001) proposed that, whereas item familiarity can be represented adequately with one Gaussian distribution, associative information may best be captured with a "some-or-none" variable, or two finite mixture distributions that correspond to items with and without any associative information, respectively (for a comparison with other related models, see Macho, 2004).

In terms of the psychological nature of the processes at work, an important aspect of the current data concerns the potential role of recollection of episodic detail for the famous names presented. In our analyses, we tested whether a high-threshold parameter $(T)$, identical to the one that indexes recollection in the DPSD model, significantly improved the model fit once the mixture parameter had been introduced. We found no evidence for improved fit with such a recollection parameter. This finding appears to be inconsistent with recent work, not based on ROC methodology, that points to involvement of recollection processes in the processing of famous names. Past studies have shown that some famous names are particularly likely to elicit recall of a specific prior personal experience pertaining to the celebrity, which gives these names autobiographical significance compared with other famous names (Piolino et al., 2007; Westmacott \& Moscovitch, 2003). For example, for John Lennon, a participant may be able to recall a particular experience of watching him on television or of hearing about his assassination. Westmacott and Moscovitch (2003) reported that famous names with autobiographical significance are associated with processing benefits on a number of cognitive tasks, including dichotomous fame judgments. These findings, however, were based on the use of famous names that were very well known to participants. This characteristic of their stimulus set is reflected in the fact that discrimination performance, again unlike in the current data, was almost perfect in that study. Thus, it is possible that recollection only contributes to discrimination when the famous names are associated with more familiarity and/or semantic knowledge than what would be present for the moderately famous names used in our study. In addition, the influence of autobiographical significance was reflected in changes in reaction times for identified names, rather than changes in confidence in the context of a detection model. Overall, it appears that recollection may not contribute to fame judgments under all circumstances. However, given the methodological differences between the small number of studies that have examined the issue, further work is clearly necessary to obtain a better understanding of the role of recollection in fame judgments and other memory decisions traditionally related to semantic memory.
Another issue for future investigations is to determine whether and how the graded memory evidence for famous names that we isolate here relates to the presence and degree of available semantic knowledge. In computational implementations of recognition processes for concepts (rather than people), such as the source of activation confusion model, familiarity is reflected in variable degrees of activation at a specific semantic node that pertains to the concept in question (Diana et al., 2006; Reder et al., 2000). In global matching models, graded recognition judgments have been assumed to be sensitive to the summed similarity of the test probe to all of the study items (Clark \& Gronlund, 1996). Put into the context of famous-name recognition, participants' graded judgments may be a direct reflection of the degree of relevant semantic knowledge that is available to them and may be partially determined by the semantic similarity of the name in question to all other famous names that participants know. On the other hand, research has shown that names can appear famous to participants simply because they were encountered recently, irrespective of any semantic knowledge participants may have (i.e., the false fame effect; Jacoby, Kelley, Brown, \& Jasechko, 1989). Considered together, the evidence currently available does not allow for any firm conclusion as to the specific role of semantic knowledge in graded fame judgments. Regardless, the current mathematical characterization provides a starting point for understanding the nature of the memory signal that allows them to be discriminated from fictional names.

Finally, our results have relevance with respect to the degree to which recognition-memory experiments can be considered an appropriate model of recognition experiences outside the laboratory, which are not tied to one controlled study episode. That recognition memory can provide a suitable model for recognition decisions outside the laboratory is often assumed implicitly in research based on the use of recognition-memory tasks with experimentally controlled study phases. The widely used remember-know paradigm, for example, involves instructions that require participants to use "know" for recognition experiences that have subjective similarity to perceiving a person outside the laboratory as familiar (e.g., Gardiner, Ramponi, \& Richardson-Klavehn, 1998). Another particularly influential example of using real-life recognition experiences to motivate research that employs the recognitionmemory paradigm is the butcher-on-the-bus phenomenon first described by Mandler (1980). This phenomenon refers to a subjective experience in which someone who is known in one particular context can appear particularly familiar when encountered in a different context without initial identification. Again, this can be seen as an example of a recognition experience that would typically hinge on lifetime experience with multiple encounters, rather than one specific episode, as would be modeled in the recognition-memory paradigm. Although there appear to be many differences between recognition judgments based on lifetime experience, such as the fame judgments employed here, and typical item-recognition memory tasks, the current work shows that recognition decisions based on lifetime exposure and those based on an experimentally controlled study phase are similar in at least one important way: They can both be well described by invoking graded evidence in the context of signal-detection mechanisms. 


\section{References}

Akaike, H. (1974). A new look at the statistical model identification. IEEE Transactions on Automatic Control, 19, 716-723. doi:10.1109/ TAC.1974.1100705

Atkinson, R. C., \& Juola, J. F. (1974). Seach and decision processes in recognition memory. In D. H. Krantz, R. C. Atkinson, \& P. Suppes (Eds.), Contemporary developments in mathematical psychology (pp. 239-289). San Francisco, CA: Freeman.

Bröder, A., \& Schütz, J. (2009). Recognition ROCs are curvilinear-or are they? On premature arguments against the two-high-threshold model of recognition. Journal of Experimental Psychology: Learning, Memory, and Cognition, 35, 587-606. doi:10.1037/a0015279

Brown, S., \& Steyvers, M. (2005). The dynamics of experimentally induced criterion shifts. Journal of Experimental Psychology: Learning, Memory, and Cognition, 31, 587-599. doi:10.1037/0278-7393.31.4.587

Clark, S., \& Gronlund, S. (1996). Global matching models of recognition memory: How the models match the data. Psychonomic Bulletin \& Review, 3, 37-60. doi:10.3758/BF03210740

DeCarlo, L. T. (2002). Signal detection theory with finite mixture distributions: Theoretical developments with applications to recognition memory. Psychological Review, 109, 710-721. doi:10.1037/0033295X.109.4.710

DeCarlo, L. T. (2003). An application of signal detection theory with finite mixture distributions to source discrimination. Journal of Experimental Psychology: Learning, Memory, and Cognition, 29, 767-778. doi: 10.1037/0278-7393.29.5.767

DeCarlo, L. T. (2008). Process dissociation and mixture signal detection theory. Journal of Experimental Psychology: Learning, Memory, and Cognition, 34, 1565-1572. doi:10.1037/a0013081

Diana, R., Reder, L., Arndt, J., \& Park, H. (2006). Models of recognition: A review of arguments in favor of a dual-process account. Psychonomic Bulletin \& Review, 13, 1-21. doi:10.3758/BF03193807

Egan, J. P. (1958). Recognition memory and the operating characteristic (Technical Report AFCRC-TN-58-51). Bloomington, IN: Hearing and Communication Laboratory, Indiana University.

Erdfelder, E., Küpper-Tetzel, C., \& Mattern, S. (2011). Threshold models of recognition and the recognition heuristic. Judgment and Decision Making, 6, 7-22.

Fortin, N., Wright, S., \& Eichenbaum, H. (2004). Recollection-like memory retrieval in rats is dependent on the hippocampus. Nature, 431, 188-191. doi:10.1038/nature02853

Gardiner, J. M., Ramponi, C., \& Richardson-Klavehn, A. (1998). Experiences of remembering, knowing, and guessing. Consciousness and Cognition, 7, 1-26. doi:10.1006/ccog.1997.0321

Green, D. M., \& Swets, J. A. (1966). Signal detection theory and psychophysics. New York, NY: Wiley.

Hilford, A., Glanzer, M., Kim, K., \& DeCarlo, L. T. (2002). Regularities of source recognition: ROC analysis. Journal of Experimental Psychology: General, 131, 494-510. doi:10.1037/0096-3445.131.4.494

Jacobs, A. M., Graf, R., \& Kinder, A. (2003). Receiver operating characteristics in the lexical decision task: Evidence for a simple signaldetection process simulated by the multiple read-out model. Journal of Experimental Psychology: Learning, Memory, and Cognition, 29, 481488. doi:10.1037/0278-7393.29.3.481

Jacoby, L. L., Kelley, C., Brown, J., \& Jasechko, J. (1989). Becoming famous overnight: Limits on the ability to avoid unconscious influences of the past. Journal of Personality and Social Psychology, 56, 326-338. doi:10.1037/0022-3514.56.3.326

Kelley, R., \& Wixted, J. (2001). On the nature of associative information in recognition memory. Journal of Experimental Psychology: Learning, Memory, and Cognition, 27, 701-722. doi:10.1037/0278-7393.27.3.701

Kinder, A., \& Assmann, A. (2000). Learning artificial grammars: No evidence for the acquisition of rules. Memory \& Cognition, 28, 13211332. doi:10.3758/BF03211833
Krantz, D. (1969). Threshold theories of signal detection. Psychological Review, 76, 308-324. doi:10.1037/h0027238

Larkin, W. D. (1965). Rating scales in detection experiments. Journal of the Acoustical Society of America, 37, 748-749. doi:10.1121/1.1909411

Luce, R. D. (1963). A threshold theory for simple detection experiments. Psychological Review, 70, 61-79. doi:10.1037/h0039723

Macho, S. (2004). Modeling associative recognition: A comparison of two-high-threshold, two-high-threshold signal detection, and mixture distribution models. Journal of Experimental Psychology: Learning, Memory, and Cognition, 30, 83-97. doi:10.1037/0278-7393.30.1.83

Macmillan, N., \& Creelman, D. (2005). Detection theory: A user's guide (2nd ed.). New York, NY: Psychology Press.

Macmillan, N., Rotello, C., \& Verde, M. (2005). On the importance of models in interpreting remember-know experiments: Comments on Gardiner et al.'s (2002) meta-analysis. Memory, 13, 607-621. doi:10.1080/ 09658210444000269

Malmberg, K. (2002). On the form of ROCs constructed from confidence ratings. Journal of Experimental Psychology: Learning, Memory, and Cognition, 28, 380-387. doi:10.1037/0278-7393.28.2.380

Malmberg, K., \& Xu, J. (2006). The influence of averaging and noisy decision strategies on the recognition memory ROC. Psychonomic Bulletin \& Review, 13, 99-105. doi:10.3758/BF03193819

Mandler, G. (1980). Recognizing: The judgment of previous occurrence. Psychological Review, 87, 252-271. doi:10.1037/0033-295X.87.3.252

Ogilvie, J., \& Creelman, C. (1968). Maximum-likelihood estimation of receiver operating characteristic curve parameters. Journal of Mathematical Psychology, 5, 377-391. doi:10.1016/0022-2496(68)90083-7

Onyper, S. V., Zhang, Y. X., \& Howard, M. W. (2010). Some-or-none recollection: Evidence from item and source memory. Journal of Experimental Psychology: General, 139, 341-364. doi:10.1037/a0018926

Paap, K. R., Chun, E., \& Vonnahme, P. (1999). Discrete threshold versus continuous strength models of perceptual recognition. Canadian Journal of Experimental Psychology, 53, 277-293. doi:10.1037/h0087316

Parks, C., \& Yonelinas, A. (2007). Moving beyond pure signal-detection models: Comment on Wixted (2007). Psychological Review, 114, 188 202.

Piolino, P., Lamidey, V., Desgranges, B., \& Eustache, F. (2007). The semantic and episodic subcomponents of famous person knowledge: Dissociation in healthy subjects. Neuropsychology, 21, 122-135. doi: 10.1037/0894-4105.21.1.122

Ratcliff, R., Sheu, C. F., \& Gronlund, S. D. (1992). Testing global memory models using ROC curves. Psychological Review, 99, 518-535. doi: 10.1037/0033-295X.99.3.518

Reder, L. M., Nhouyvanisvong, A., Schunn, C. D., Ayers, M. S., Angstadt, P., \& Hiraki, K. (2000). A mechanistic account of the mirror effect for word frequency: A computational model of remember-know judgments in a continuous recognition paradigm. Journal of Experimental Psychology: Learning, Memory, and Cognition, 26, 294-320. doi:10.1037/ 0278-7393.26.2.294

Schwarz, G. E. (1978). Estimating the dimension of a model. Annals of Statistics, 6, 461-464. doi:10.1214/aos/1176344136

Self, S. G., \& Liang, K. Y. (1987). Asymptotic properties of maximum likelihood estimators and likelihood ratio tests under nonstandard conditions. Journal of the American Statistical Association, 82, 605-610. doi: $10.2307 / 2289471$

Sherman, S. J., Atri, A., Hasselmo, M. E., Stern, C. E., \& Howard, M. W. (2003). Scopolamine impairs human recognition memory: Data and modeling. Behavioral Neuroscience, 117, 526-539. doi:10.1037/07357044.117.3.526

Snodgrass, J., \& Corwin, J. (1988). Pragmatics of measuring recognition memory: Applications to dementia and amnesia. Journal of Experimental Psychology: General, 117, 34-50. doi:10.1037/0096-3445.117.1.34

Sokal, R. R., \& Rohlf, F. J. (1994). Biometry: The principles and practice of statistics in biological research (3rd ed.). New York, NY: Freeman. 
Stenberg, G., Hellman, J., \& Johansson, M. (2008). The memorability of names and the divergent effects of prior experience. European Journal of Cognitive Psychology, 20, 312-345. doi:10.1080/09541440701398724

Westmacott, R., \& Moscovitch, M. (2003). The contribution of autobiographical significance to semantic memory. Memory \& Cognition, 31, 761-774. doi:10.3758/BF03196114

Wickens, T. D. (2002). Elementary signal detection theory. New York, NY: Oxford University Press.

Wixted, J. (2007a). Dual-process theory and signal-detection theory of recognition memory. Psychological Review, 114, 152-176. doi:10.1037/ 0033-295X.114.1.152

Wixted, J. (2007b). Spotlighting the probative findings: Reply to Parks and Yonelinas Psychological Review, 114, 203. doi:10.1037/0033295X.114.1.203

Yonelinas, A. P. (1994). Receiver-operating characteristics in recognition memory: Evidence for a dual-process model. Journal of Experimental Psychology: Learning, Memory, and Cognition, 20, 1341-1354. doi: 10.1037/0278-7393.20.6.1341

Yonelinas, A. P. (1999). The contribution of recollection and familiarity to recognition and source-memory judgments: A formal dual-process model and an analysis of receiver operating characteristics. Journal of Experimental Psychology: Learning, Memory, and Cognition, 25, 14151434. doi:10.1037/0278-7393.25.6.1415

Yonelinas, A. P., \& Parks, C. M. (2007). Receiver operating characteristics (ROCs) in recognition memory: A review. Psychological Bulletin, 133, 800-832. doi:10.1037/0033-2909.133.5.800

Received November 8, 2010 Revision received June 9, 2011

Accepted June 13, 2011

\section{Call for Nominations}

The Publications and Communications (P\&C) Board of the American Psychological Association has opened nominations for the editorships of Journal of Experimental Psychology: Animal Behavior Processes, Journal of Experimental Psychology: Applied, Neuropsychology, and Psychological Methods for the years 2014-2019. Anthony Dickinson, PhD, Wendy A. Rogers, $\mathrm{PhD}$, Stephen M. Rao, $\mathrm{PhD}$, and Scott E. Maxwell, $\mathrm{PhD}$, respectively, are the incumbent editors.

Candidates should be members of APA and should be available to start receiving manuscripts in early 2013 to prepare for issues published in 2014. Please note that the P\&C Board encourages participation by members of underrepresented groups in the publication process and would particularly welcome such nominees. Self-nominations are also encouraged.

Search chairs have been appointed as follows:

- Journal of Experimental Psychology: Animal Behavior Processes, John Disterhoft, PhD, and Linda Spear, PhD

- Journal of Experimental Psychology: Applied, Jennifer Crocker, PhD, and Lillian ComasDiaz, PhD

- Neuropsychology, Norman Abeles, $\mathrm{PhD}$

- Psychological Methods, Neal Schmitt, PhD

Candidates should be nominated by accessing APA's EditorQuest site on the Web. Using your Web browser, go to http://editorquest.apa.org. On the Home menu on the left, find "Guests." Next, click on the link "Submit a Nomination," enter your nominee's information, and click "Submit."

Prepared statements of one page or less in support of a nominee can also be submitted by e-mail to Sarah Wiederkehr, P\&C Board Search Liaison, at swiederkehr@apa.org.

Deadline for accepting nominations is January 10, 2012, when reviews will begin. 